\title{
Global Gene Expression Divergence in Spontaneous Mutation Accumulation Lines of Caenorhabditis elegans under Varying Efficiency of Selection
}

\author{
Thaddeus C. Deiss ${ }^{1}$ \\ Anke Konrad ${ }^{1,2}$ \\ Ulfar Bergthorsson ${ }^{1 \dagger}$ \\ Vaishali Katju ${ }^{1 \dagger}$
}

\author{
${ }^{1}$ Department of Veterinary Integrative Biosciences, College of Veterinary Medicine and \\ Biomedical Sciences, Texas A\&M University, College Station, TX 77845, USA \\ ${ }^{2} \mathrm{CE} 3 \mathrm{C}$ - Centre for Ecology, Evolution and Environmental Changes, Faculdade de \\ Ciências da Universidade de Lisboa, Campo Grande, 1749-016 Lisboa, Portugal \\ $\dagger$ Joint corresponding authors: \\ vkatju@cvm.tamu.edu \\ ubergthorsson@cvm.tamu.edu
}

Keywords:

Caenorhabditis elegans | gene expression divergence | mutation accumulation | selection | genetic drift | RNA-Seq

Running Title:

Expression divergence during mutation accumulation 


\section{ABSTRACT}

To ascertain the effect of relaxation of selection on global gene expression, Caenorhabditis elegans mutation accumulation (MA) lines were propagated under varying degrees of efficiency of selection determined by their different population sizes $\left(N=1,10\right.$ and 100). Both the mutational variance $\left(V_{m}\right)$, and the residual variance $\left(V_{r}\right)$ were greatest in MA lines with the lowest efficiency of natural selection. The results suggest that gene expression is under strong balancing selection. Furthermore, mutations resulting in increased transcriptional noise or sensitivity to microenvironmental variation accumulate most under extreme genetic drift. In contrast, the $V_{m} / V_{r}$ ratio was lowest in the $N=1$ lines. Chromatin domains associated with broad gene silencing and active transcription exhibited the greatest and the smallest increase in transcriptional variation, respectively. Furthermore, the preponderance of overexpressed genes was especially pronounced in mitochondrial respiration, stress response, and immune system pathways, especially in low fitness $N=1$ lines. 


\section{INTRODUCTION}

Experimental investigations into the rate of spontaneous mutations, along with

4 their molecular and phenotypic consequences, are central to the study of evolution and

5 biology, with important implications for human health including disease susceptibility

6 and inherited genetic disorders. Regulation of gene expression has long been understood

7 to play a considerable role in evolution and most phenotypic evolution, both intraspecific

8 and interspecific, is likely due to changes in gene expression rather than changes in

9 protein structure (Britten and Davidson 1969; King and Wilson 1975; Stern 1998;

10 Wittkopp et al. 2003; Wray et al. 2003; Abzhanov et al. 2004; Fay et al. 2004; Shapiro et

11 al. 2004; Gompel et al. 2005; Gilad et al. 2006; Fay and Wittkopp 2008; Loehlin et al.

12 2019).

The phenotypic consequences of spontaneous mutations in their broadest sense,

15 including behavioral, biochemical and physiological traits, are realized and constrained

16 by the rules of gene expression and development. Characterizing patterns of gene

17 expression divergence in natural and experimental populations is therefore an important

18 step towards understanding how mutations and epimutations exert their influence on the

19 evolution of gene expression. Furthermore, questions regarding the relative importance of

20 natural selection versus genetic drift in shaping the evolution of expression require

21 knowledge about the effects of spontaneous mutations on gene expression. The influence

22 of these two major evolutionary forces, drift and selection, can be investigated by

23 comparing populations or experimental laboratory lines under minimal selection (strong

24 genetic drift) with their counterparts that are subject to strong intensity of selection (e.g.

25 natural populations) (Denver et al. 2005; Rifkin et al. 2005; McGuigan et al. 2014b;

26 Huang et al. 2016). Moreover, just as genes that differ markedly in their rates of sequence

27 evolution have provided evidence for selective constraints and positive selection, genes

28 may also exhibit differential capacity to evolve at the transcriptional level (Rifkin et al.

29 2005; Landry et al. 2007). In a related vein, gene expression divergence may also vary

30 across developmental stages, exhibiting highly constrained patterns in some stages

31 (developmental constraints) relative to others (Zalts and Yanai 2017). 
Additional questions of interest relate to the nature of genetic change driving

34 divergence of gene expression. Given the considerable variation in genome organization

35 of different groups of organisms, how might a species' prevailing genomic and genetic

36 architecture impinge on the evolution of its transcriptome? The genomes of eukaryotic

37 species are highly variable in size, chromosomal organization, and chromatin state, and

38 can comprise large expanses of repetitive, gene-poor regions of low complexity as well as

39 a high incidence of selfish genetic elements. Additionally, there exists considerable

40 variation in recombination frequency which, in conjunction with selection, can further

41 influence the patterns of nucleotide variation, and in turn effect differential divergence at

42 the transcriptional level. Investigating how these different properties of spontaneous

43 mutations and genome architecture influence the evolution of gene expression is of

44 fundamental importance.

In order to determine the effective role of mutation in shaping expression profiles,

47 many studies opt to negate the force of selection acting constitutively within natural

48 populations. Mutation accumulation (MA henceforth) is a commonly implemented

49 experimental design to isolate the effect of mutation from selective pressures (Halligan

50 and Keightley 2009; Katju and Bergthorsson 2019). In an MA experiment, multiple

51 replicate lines derived from an inbred ancestral stock population are allowed to evolve

52 independently of one another under conditions of extreme bottlenecking each generation.

53 The repeated bottlenecks severely diminish the efficacy of natural selection, promoting

54 evolutionary divergence due to the accumulation of deleterious mutations by random

55 genetic drift. While spontaneous MA experiments provide a powerful framework to

56 investigate divergence in global transcription profiles due to accumulated genetic changes

57 in the near absence of selection, only a limited set have been analyzed at the

58 transcriptional level. These studies have determined global mutational variance $\left(V_{m}\right)$ in a

59 few eukaryotic species comprising nematodes (Denver et al. 2005), flies (Rifkin et al.

60 2005; McGuigan et al. 2014a, 2014b; Huang et al. 2016), and yeast (Landry et al. 2007).

61 More recent studies have focused on the transcriptional profiles of copy-number variants

62 (CNVs) and transposable elements in Caenorhabditis elegans following MA (Konrad et 
63 al. 2018; Bergthorsson et al. 2020). Some initial conclusions from these studies suggest

64 that gene expression divergence is under strong stabilizing selection. But despite strong

65 selection, there is also a correlation between transcriptional effects of spontaneous

66 mutations and divergence between closely-related species, suggesting that mutational

67 input exerts a significant influence on the patterns of gene expression divergence (Rifkin

68 et al. 2005).

Although MA experiments are canonically designed to negate the effect of

72 bottlenecks, this study aimed to identify trends in expression variability and divergence

73 within lines subjected to varying efficacy of selection (Konrad et al. 2017, 2018, 2019).

74 Experimental MA lines were maintained at three population sizes of $N=1,10$, and 100

75 individuals per generation as outlined in Katju et al. $(2015,2018)$, thereby enabling

76 comparison of transcript abundance between populations evolved under the near absence

77 of selection $(N=1)$ versus those subject to increasing efficacy of selection $(N=10$, and

78 100). A comparison of the mutational and residual variance between these different

79 population size treatments can be used to investigate the effects of selection and drift on

80 both the mutational heritability and plasticity in gene expression.

\section{METHODS}

Experimental study system and mutation accumulation design

We conducted a long-term spontaneous MA experiment in C. elegans comprising

8735 populations maintained in parallel at varying population size treatments of $N=1,10$,

88 and 100 hermaphrodites $\left(N_{\mathrm{e}}=1,5,50\right.$ individuals, respectively) per generation over four

89 and a half years and spanning 409 MA generations (Katju et al. 2015, 2018). All

90 experimental lines of the MA experiment were established from the descendants of a

91 single wild-type Bristol (N2) hermaphrodite originally isolated as a virgin L4 larva

92 (Supplemental Figure S1A) with excess animals cryogenically preserved at $-86^{\circ} \mathrm{C}$ as

93 ancestral controls. The MA experiment was conducted under benign laboratory 
94 conditions. Additional details on methodology are provided in Katju et al. (2015). Stocks

95 of the MA lines were cryogenically preserved every 50-100 MA generations during the

96 course of the experiment.

97

98 Theoretical underpinnings

99

100

Mutational fitness effects can range continuously from lethal to deleterious to

101 neutral to beneficial. Mutations in small populations are governed by genetic drift, with

102 beneficial mutations lost and detrimental mutations fixed randomly within the population.

103 However, as population size increases, natural selection steadily supplants genetic drift in

104 dictating which mutations are lost versus fixed. Hence, the fate of a novel mutation, be it

105 loss or fixation, is dependent upon both the selection coefficient $(s)$ and effective

106 population size $\left(N_{e}\right)$. The mutational dynamics for sexually reproducing diploids has been

107 shown to be dominated by random drift when $|s|<<1 / 2 N_{e}$ (Kimura 1962). In contrast the

108 dynamics for mutations with $|s|>>1 / 2 N_{e}$ are dictated by natural selection. Complete

109 inbreeding of obligate self-fertilizing hermaphrodites, such as C. elegans, results in a

$11050 \%$ reduction in $N_{e}$ relative to the census population size $(N)$ (Crow and Kimura 1970;

111 Pollak 1987; Charlesworth 2009). This means that the effective population sizes for our

112 experimental lines of $N=1,10$ and 100 individual(s) correspond to $N_{e}=1,5$ and 50

113 individuals respectively. The fate of mutations in our MA lines of $N=1,10$ and 100 is

114 expected to be governed by genetic drift when selection coefficients are less than $0.5,0.1$

115 and 0.01 respectively. The demarcation between the behavior of mutations with $2 N_{e}<1$,

116 which should be dominated by genetic drift and $2 N_{e}>1$, which are under greater

117 influence of natural selection, is not sharply defined and some mutations with a larger

118 fitness cost than $1 / 2 N_{e}$ could be fixed by genetic drift. Nonetheless, the differences in

119 populations size in these MA experiments alters the relative importance of genetic drift

120 versus natural selection in the fixation or loss of mutations, with genetic drift having the

121 greatest influence in $N=1$ lines and diminishing in strength with increasing population

122 size.

123

124

$R N A$ extraction, library preparation and $R N A-S e q$ 
One individual was isolated from each of $17 N=1$ lines, while two and three individuals were isolated from MA lines of size $N=10$ (10 lines), and $N=100$ (five

127 lines), respectively. Given that the $N=1 \mathrm{MA}$ lines are expected to be genetically identical

128 and homozygous due to self-fertilization for several hundred generations and extreme

129 bottleneck size, we only isolated a single individual per $N=1$ MA line for RNA-

130 sequencing. MA lines maintained at larger population sizes were expected to harbor

131 greater standing genetic variation. Hence, we isolated two and three randomly picked

132 individuals from each $N=10$ line and $N=100$ line, respectively. Additionally, five

133 randomly picked individuals were isolated from the pre-MA ancestral control. The

134 isolated individuals were sequestered on to NGM plates containing OP50 lawns and kept

135 at $20^{\circ} \mathrm{C}$. For each of these 57 individuals, three offspring at the L4 larval stage were

136 isolated from the $\mathrm{F}_{1}$ generation to serve as biological replicates in the expression analysis

137 (Supplemental Figure S1B), yielding a total of 171 samples for tissue collection and

138 RNA sequencing at the L1 larval stage. All 171 sample populations were maintained for

139 a few generations until enough worms were available for RNA extraction. Synchronized

140 populations of L1 larvae were generated through the collection of gravid eggs from adults

141 using a standard bleaching protocol and plated on unseeded NGM plates. Eggs from the

142 synchronized populations were allowed 12 hours to hatch and L1 larvae were collected

143 for total RNA extraction. Total RNA isolation was performed using the Qiagen RNeasy

144 kit (Cat no. 74106) according to manufacturer's protocol with addition of a glass bead

145 beating step to aid in nematode lysis. Quantification and assessment of RNA integrity

146 was determined via Qubit 3.0 Fluorometer, HS RNA assay (Cat no. Q32852), and

147 Agilent TapeStation, HS RNA assay (Cat no. 5067-5580), respectively. Illumina libraries

148 were constructed from $0.5 \mu \mathrm{g}$ of total RNA with the Illumina Truseq stranded mRNA kit

149 and adapter indexes (Cat no. 20020594/20019892) according to manufacturer protocols.

150 Libraries were pooled at a concentration of $5 \mathrm{nM}$ for paired-end sequencing on the

151 Illumina HiSeq4000 platform $(2 \times 150 \mathrm{bp})$ at the Texas A\&M Genomics and

152 Bioinformatics Service Center or Illumina Novaseq6000 $(2 \times 150 \mathrm{bp})$ at the North Texas

153 Genome Center at the University of Texas at Arlington. Sequence data are deposited to

154 the NCBI database with the accession number for the Bioproject PRJNA448413. 

alignment software (Dobin et al. 2013) using default settings and the C. elegans WS274 genome sequence and annotation files as references for mapping and transcript quantification, respectively. Gene specific read counts for each biological replicate were used to construct a raw matrix for all downstream analyses. Transcripts per million (TPM) were calculated gene-wise, using the longest transcript isoform for alternatively spliced genes, for each sample using the following equation:

$$
T P M=\frac{\text { bases mapped to gene }}{\text { bases in longest isoform }} \times \frac{1}{\text { bases mapped in sample library }} \times 10^{6}
$$

Genes with less than an average three TPM cutoff across all samples were excluded in all subsequent analyses. The filtered dataset contained 11,686 genes on which all downstream analyses were performed. Unless otherwise specified, the ratio of the MA line to the ancestral TPM values were $\log _{2}$-transformed prior to all downstream analyses to facilitates comparisons to previously reported microarray results.

174 design matrix built to compare the individual biological replicates for MA lines to the 175 ancestral control biological replicates. The resulting $p$-values were adjusted using the

176 Benjamini-Hochberg FDR correction for false discovery rate in multiple comparison

177 testing. Differential expression analysis results were filtered with thresholds for adjusted

$178 p$-value (adjusted $p \leq 0.01)$ and magnitude $\left(20 \%\right.$ change over ancestral $\mathrm{N} 2$ control, $\log _{2}$ -

179 fold change $=0.26$ ). In summation the differential expression analysis returned

180 comparisons for $3 \times 17$ samples from the $N=1$ populations, $3 \times 20$ for $N=10$, and $3 \times 15$

181 for $N=100$ to the ancestral control.

184 differential expression gene lists with the "clusterProfiler" package (Yu et al. 2012) in R.

185 The background gene list used for significance testing was composed of genes that were 
186 found to be differentially expressed between any MA sample and the ancestor regardless

187 of population size. The minimum number of background genes per category was 50 while

188 the number of differentially expressed genes per experimental category was set at five. A

189 Benjamini-Hochberg adjusted $p$-value of 0.05 was used as a significance cutoff for GO

190 category enrichment. A Wilcoxon-Signed Rank test was employed to determine

191 significant differences in the number of underexpressed to overexpressed genes for all

192 experimental populations. Population-specific differences in the distributions of

193 overexpressed, underexpressed, and overexpressed:underexpressed genes were

194 determined using Kruskal-Wallis tests.

\section{Estimation of the Mutational and Residual Variance}

Estimates of residual variance $\left(V_{r}\right)$ and mutational variance $\left(V_{m}\right)$ were calculated in R (R Core Team 2014) using the model below for each gene allowing for populationspecific across-line variance and line-specific residual variances:

$$
\left.y_{i j k}=\mu+\text { Pop.Trt. }{ }_{. i}+\text { MALine (Pop.Trt. }\right)_{j(i)}+\varepsilon_{i j k}
$$

202 where $y_{i j k}$ is the gene-wise $\log _{2}$-ratio of the MA sample to average ancestral value for

203 each MA sample $k$, of each MA line $j$, evolved under population size treatment $i$.

204 Mutational variance $\left(V_{m}\right)$ was calculated as the variance across experimental MA lines for

205 each population size treatment divided by $2 \times$ the average number of generations per line

206 for each population. This calculation yielded a single $V_{m}$ value per gene for each

207 population size treatment $(N=1,10$ and 100) which was then used to perform Kruskal-

208 Wallis tests for differences in the distribution of $V_{m}$ between the three population size

209 treatments.

The residual variance $\left(V_{r}\right)$ was calculated gene-wise for the three biological

212 replicates of each genotype via two different methods depending on the downstream

213 statistical comparisons. The first calculation used raw TPM values to compare $V_{r}$ between

214 MA lines and the ancestral control. The second approach used the $\log _{2}$-ratios of MA line

215 replicates to ancestral control to determine $V_{r}$ in the same manner as was used for

216 calculating $V_{m}$. Both of these calculations yielded gene-specific $V_{r}$ estimates for each MA 
217 lines in the study. A Kruskal-Wallis test was used to investigate differences in the

218 distribution of $V_{r}$ between the three MA treatments $(N=1,10$, and 100) and the ancestral

219 control). Following a significant Kruskal-Wallis test, a Dunn's Multiple Comparison Test

220 test with a Benjamin-Hochberg stepwise adjustment was used to conduct multiple pair-

221 wise comparisons for median difference. Each of the plate used for extracting RNA

222 contained several thousand nematodes. Because the number of worms per RNA

223 extraction for each biological replicate was unknown, we did not estimate the mutational

224 heritability $\left(h_{m}^{2}\right)$. Under the assumption that there were no systematic differences in

225 sample size between MA lines, we calculated the $V_{m} / V_{r}$ ratio for different population size

226 treatments in lieu of $h_{m}^{2}$.

Histone Modification Datasets and Analysis

Genes were additionally assigned to one or more histone modification categories

231 based on a previous C. elegans ChIP-seq expression study (Liu et al. 2011). Publicly

232 available ChIP-Seq datasets were downloaded from the modMine repository

233 (http://intermine.modencode.org) (Contrino et al. 2012). (Supplemental Table S1).

234 Datasets lacking pre-analyzed coordinates were downloaded in wiggle (WIG) format for

235 analysis of peaks using the MACS2 software package (Zhang et al. 2008). A minimum

236 interval of $1000 \mathrm{bp}$ was used to determine histone associated genomic tracks with the

237 default peak cutoff suggested by the cutoff-analysis tool. Since our samples were L1

238 larvae and publicly available datasets were mostly composed of early embryo or L3

239 larvae, we only assigned genes to a category if there was a consensus amongst all ChIP-

240 Seq datasets for a given modification. For example, genes were only assigned to the

$241 H 3 K 27 m e 1$ category if they were found within a positive track for all three publicly

242 available CHiP-Seq datasets.

244 Statistical Analyses

All calculations and statistical tests were performed in $\mathrm{R}$ with graphs produced

247 using the ggplot2 package (Wickham 2016). 


\section{RESULTS}

We employed a spontaneous MA experiment design in C. elegans comprising

252 three population size treatments of $N=1,10,100$ individuals all descended from a single

253 N2 worm ancestor (Katju et al. 2015, 2018). The MA lines of differing population sizes

254 were subjected to bottlenecks for $>400$ consecutive generations, allowing us to jointly

255 assess the genome-wide, molecular consequences of different mutation classes (in both

256 the mitochondrial and nuclear genomes) under conditions of neutrality and with

257 increasing intensity of selection (Konrad et al. 2017, 2018, 2019). In two preceding

258 studies, we have assessed the transcriptional consequences of copy-number variants

259 (gene duplications and deletions) and transposable elements (TEs) (Konrad et al. 2018;

260 Bergthorsson et al. 2020). In both these studies, the $N=1$ lines that were subject to the

261 greatest intensity of genetic drift displayed the greatest increase in transcriptional activity

262 in gene duplications and TEs relative to the ancestral control. In this study, we further

263 extend the transcriptional analyses of these evolved experimental lines to investigate the

264 divergence in global transcription profiles due to the input of other classes of new

265 mutational variants (excluding gene copy-number changes and TEs), both under minimal

266 influence of purifying selection $(N=1$ lines) and with incrementally increasing efficacy of

267 selection $(N=10$ and 100 lines $)$.

Greater mutational and residual variance at small population size

We have previously reported that gene copy-number variants (CNVs) are more

272 common in the $N=1 \mathrm{MA}$ line, and that the increase in transcript abundance associated

273 with gene duplications is also greater in the $N=1 \mathrm{MA}$ lines compared to the $N=10$ and

$274 N=100$ MA lines (Konrad et al. 2018). In addition, TE transcription exhibits a negative

275 relationship with population size in MA lines (Bergthorsson et al. 2020). The results

276 presented here were calculated after filtering out genes that were known to be duplicated

277 or deleted (CNVs), as well as annotated transposable elements (TEs). The mutational

278 variance, $V_{m}$, is a fundamental parameter in models of quantitative traits, and is defined as 
279 the rate of increase in genetic variation per generation due to mutation. $V_{m}$ was estimated

280 as the increase in the variance between MA lines within a particular population

281 bottleneck size standardized by $2 \times$ the average number of generations. The median $V_{m}$

282 values for transcript abundance after removing CNVs were $1.11,0.89$, and $0.85 \times 10^{-4}$ per

283 gene for the $N=1,10$ and 100 lines, respectively (Figure 1A; Kruskal-Wallis: $\chi^{2}=$

$\left.284447.82, p=5.71 \times 10^{-89}\right)$. This is consistent with the hypothesis that transcript abundance

285 is under selection and that strong genetic drift associated with smaller population sizes

286 results in the accumulation of increased deleterious variation in expression.

If relaxation of natural selection results in increased transcriptional dysregulation, the within-line variance in transcript abundance of individual genes (residual variance, $V_{r}$ ) would be higher in the $N=1$ relative to the $N=10$ and $N=100$ MA lines. As above, CNVs and TEs were removed prior to the analysis. There was a significant difference in the median TPM $V_{r}$ values of 5.89, 3.92, and 4.59 for the $N=1,10$ and 100 lines,

294 population size treatments comprising our MA experiment, the $N=1$ lines were observed

295 to have significantly higher $V_{r}$ values than the $N=10$ or 100 MA lines (Figure 1B).

296 Extreme relaxation of selection, as in the case of the $N=1$ MA lines, increases variation

297 in transcript abundance among replicates within lines relative to the larger population size

298 treatments. Furthermore, all of the MA population size treatments resulted in

299 significantly greater $V_{r}$ compared to the $\mathrm{N} 2$ ancestral control which had a median $V_{r}$ of

3002.17 (Figure 1B). This suggests that new mutations have rendered transcriptional

301 regulation of some genes less robust and perhaps more sensitive to environmental

302 variation. This contrasts with a previous $C$. elegans study in which the ancestral N2

303 control was found to have higher $V_{r}$ than the MA lines (Baer and Denver 2010).

The same features of a gene's regulatory system that contribute to greater

306 plasticity in expression, whether it is due to a response to some environmental variables

307 or stochasticity in gene regulation, may also result in greater sensitivity of expression to

308 mutations (Meiklejohn and Hartl 2002; Rifkin et al. 2005; Landry et al. 2007). Genes

309 with greater $V_{r}$ may therefore also have greater $V_{m}$, the prediction being a positive 
310 correlation between the mutational and residual variance. For this comparison, the $V_{r}$ was

311 calculated using the same approach as was employed for $V_{m}$, by generating the $\log _{2}$-ratios

312 of MA line replicates to the ancestral control. The median $V_{r}$ values using this approach

313 were $6.7,3.7$, and $4.8 \times 10^{-2}$ for the $N=1,10$, and $100 \mathrm{MA}$ lines, respectively. The

314 correlation between $V_{m}$ and $V_{r}$ is positive and highly significant (Figure 1C; Pearson's $r$

$315=0.58, p=0)$.

316

$N=1 M A$ lines exhibit the lowest $V_{m} / V_{r}$ ratio

Mutational heritability is defined as the expected heritability of an initially homozygous population following one generation of mutation (Lynch 1988; Houle et al. 1996). The ratio of the mutational variance from MA studies to the environmental variance, $V_{m} / V_{e}$, has been used to estimate the mutational heritability and in the absence 323 of experimental environmental variation, the residual variance, $V_{r}$, has sometimes been 324 used as a proxy for $V_{e}$ (Rifkin et al. 2005). Following Baer and Denver (2010), we 325 estimated $V_{r}$ from the variance in gene expression between replicate plates of worms 326 descended from the same MA genotype, wherein each plate housed several thousand L1

327 larvae. Although $V_{e}$ should be based on individual variation, we assume that the $V_{r}$

328 estimated from the variation among populations (plates) of the same genotype is a

329 function of $V_{e}$, and can be used to indicate the relative magnitude of $V_{e}$ between

330 genotypes. There were significant differences between population sizes with respect to

$331 V_{m} / V_{r}$, with the $N=1$ lines having significantly lower estimates of $V_{m} / V_{r}$ relative to the $N$

$332=10$ and 100 lines (Figure 2). The median estimates of $V_{m} / V_{r}$ are 1.68, 2.41, and 1.76

$333 \times 10^{-3}$ for the $N=1,10$, and 100 lines, respectively (Kruskal-Wallis $\chi^{2}=1225.36, p=8.25$

$\left.334 \times 10^{-267}\right)$. This suggests that the mutational heritability in the $N=1$ lines is lower than that

335 of the larger population size treatments. Despite having greater $V_{m}$, the transcriptional

336 variation generated by new mutations in the $N=1$ lines may not respond as well to

337 selection in any given environment as the transcriptional variation in the larger

338 populations, either due to greater sensitivity to environmental variation or greater

339 transcriptional noise. 
341 Reduced gene expression is a common consequence of mutation accumulation

342 but overexpression may be associated with a greater fitness cost

A moderated T-test (Smyth 2004) was performed to elucidate differential gene

345 expression profiles that developed throughout the course of mutation accumulation by

346 comparing transcript abundance of each MA genotype to that of the N2 ancestral control.

347 We defined significant change in transcript abundance as an increase or decrease of $20 \%$

348 or more, and $p \leq 0.01$. The average number of genes with significantly increased

349 transcript abundance was $626 \pm 121,367 \pm 73$ and $326 \pm 90$ for the $N=1,10$ and 100

350 MA lines, respectively (Figure 3A). The average number of genes with significantly

351 decreased transcript abundance was $1088 \pm 145,1251 \pm 276$, and $1091 \pm 354$ for $N=1$,

352 10, and $100 \mathrm{MA}$ lines, respectively (Figure 3A). Irrespective of population size

353 treatment, mutation accumulation disproportionately resulted in reduced transcription of

354 genes (Figure 3A; Wilcoxon's Signed-Ranks $T_{s}=10, p=2.0 \times 10^{-8}, n=32$ MA lines).

355 Although the number of genes with increased transcription appeared to be greater in the

$356 N=1$ lines relative to the $N>1$ lines, the difference was only marginally significant

357 (Kruskal-Wallis $\chi^{2}=3.78, p=0.051$ ). However, the ratio of up- to down-regulated genes

358 per MA line replicate was significantly different between the $N=1$ and $N>1$ lines

359 (Kruskal-Wallis $\chi^{2}=6.49, p=0.01$ ). This pattern could be suggestive of a greater

360 deleterious fitness effect of gene overexpression and a role of purifying selection in the

361 eradication of such mutations at larger population sizes. Alternatively, the association

362 between genes with increased gene expression and population size could be a

363 consequence of lower fitness or mutations causing transcriptional dysregulation in the $N$

$364=1$ lines. Additionally, we noted that the magnitude and significance of changes in

365 relative transcript abundance were also greater in the $N=1$ MA lines subject to minimal

366 efficiency of selection (Figure 3B).

368 GO enrichment analysis reveals patterns of differential expression between population 369 size treatments 
Gene ontology (GO) analyses were performed on differentially expressed genes within each line as identified in the previous section (Figure 3). A Fisher's exact test ( $p \leq$

373 0.01) was used to determine a significant enrichment with a minimum of 50 genes per

374 GO category. For population sizes of $N=1,10$, and 100, we identified significant

375 enrichment in 108, 108, and 85 biological process (BP) categories, 44, 38, and 39 cellular

376 component (CC) categories, and 40, 25, and 23 molecular function (MF) categories,

377 respectively. A complete table of enriched GO terms can be found in Supplemental

378 Table S2.

For every GO term enriched within each population size treatment, we further determined the average proportion of genes that were significantly upregulated or downregulated relative to the ancestral state within each experimental line. This analysis was performed on genes meeting significance $(p \leq 0.01)$ and $\log _{2}( \pm 0.26)$ thresholds. The

384 four GO categories with the greatest difference in the proportion of upregulated genes

385 between population size treatments were mitochondrial membrane, immune system,

386 response to biotic stimulus and unfolded protein response (Figure 4). In all cases, the 387 relative proportion of significantly upregulated genes was greater within the $N=1$ lines

388 relative to the larger populations $(N=10,100)$. Additionally, the average $\log _{2}$-fold

389 change of immune-related and mitochondrial genes was found to be negatively correlated

390 with MA line fitness (immune-related: Pearson's $r=-0.41, p=2.52 \times 10^{-3}$;

391 mitochondrial: Pearson's $r=-0.61, p=2.47 \times 10^{-6}$; Supplemental Figure S2; Katju et

392 al. 2015). The four GO categories with the greatest difference in the proportion of

393 downregulated genes between population size treatments were transcriptional regulation,

394 non-coding RNA processing, chromosome organization and ribosome biogenesis (Figure

395 4). For these categories, the $N>1$ lines had a greater number of significantly

396 downregulated genes. For comparison, Figure 4 also includes the proportion of upwardly

397 and downwardly expressed gene for two GO categories that exhibited the least difference

398 between the three population size treatments, namely enzyme inhibitor activity and

399 lysosomes.

400

401 Histone modification domains influence variation in transcript abundance 
Histone modification patterns for C. elegans were broadly divided into five

404 domains (Liu et al. 2011) associated with (i) broad gene silencing (H3K27me3), (ii)

405 repression in repetitive DNA regions (H3K9me1/me2/me3), (iii) dosage compensation

406 (H3K27me1 and H4K20me1), (iv) activation within promoter regions (H3K4me2,

407 H3K4me3, H4K8ac, H4K16ac, H4tetra-ac, and HTZ-1), and (v) active transcription

408 (H3K79me1/me2/me3 and H3K36me3). We analyzed the variation in transcript

409 abundance between genomic regions associated with different histone modification

410 domains. The average and median Coefficient of Variation (CV) per gene were

411 significantly higher within all population size treatments relative to the ancestral control

412 lines (Figure 5). Furthermore, the greatest variation was found between the $N=1$

413 lines (Figure 5). The median CV for chromatin domains associated with repression for

414 populations $N=1,10,100$ and the ancestral control were $1.7,1.42,1.55,1.03 \times 10^{-1}$

415 (Kruskal-Wallis $\chi^{2}=628.95, p=5.32 \times 10^{-136}$ ). The median CV for genes associated with

416 silencing in repetitive DNA regions was $1.34,1.11,1.24,0.95 \times 10^{-1}$ (Kruskal-Wallis $\chi^{2}=$

$417121.71, p=3.31 \times 10^{-26}$ ) for populations $N=1,10,100$ and the ancestral control,

418 respectively. For genes associated with dosage compensation, median CV values were

$4191.25,0.99,1.04,0.89 \times 10^{-1}\left(\right.$ Kruskal-Wallis $\left.\chi^{2}=228.66, p=2.70 \times 10^{-49}\right)$ for $N=1,10$,

420100 lines and the ancestral control, respectively. Variation in transcript abundance of

421 genes associated with transcriptional activation was notably lower than their repressed

422 counterparts, with a median CV of 1.18, 0.97, 1.07, $0.82 \times 10^{-1}$ (Kruskal-Wallis $\chi^{2}=$

$423686.49, p=1.78 \times 10^{-148}$ ) for $N=1,10,100$ lines and ancestral control, respectively.

424 Finally, the median CV associated with histone modification denoting constitutively

425 expressed genes was $1.15,0.92,0.98,0.87 \times 10^{-1}\left(\right.$ Kruskal-Wallis $\chi^{2}=140.39, p=3.11 \times$

$42610^{-30}$ ) for $N=1,10,100$ lines and ancestral control, respectively.

\section{DISCUSSION}

Mutation accumulation (MA) studies, originally conceived in the 1960 s to

431 indirectly estimate mutational parameters including the deleterious mutation rate from

432 fitness-related phenotypic data, have emerged as a powerful framework to directly 
433 determine the rate and spectrum of spontaneous mutations as well as their transcriptional

434 consequences. The last decade has witnessed a burgeoning of MA experiments that have

435 served to increase species representation, thereby offering insights into mutational

436 parameters in emerging model species of prokaryotes as well as new eukaryotic taxa such

437 as algae, amoeba, ciliates, crustaceans, and mammals (reviewed in Katju and

438 Bergthorsson 2019). Spontaneous MA experiments provide a powerful framework to

439 assess global transcriptional divergence due to the input of new genetic variation via

440 mutation. Yet, to date, this approach remains surprisingly underutilized. This study using

441 C. elegans represents the first attempt to analyze global transcriptional divergence in MA

442 lines using (i) next-generation sequencing technology (RNA-Seq) in conjunction with (ii)

443 a modified MA design comprising differing population size treatments to additionally

444 discern the effects of mutation and selection on transcriptional divergence.

Comparisons of transcriptional divergence within natural isolates of species

448 Rifkin et al. 2005; Hodgins-Davis et al. 2015) and MA experiments (Denver et al. 2005;

449 Rifkin et al. 2005; McGuigan et al. 2014a; Hodgins-Davis et al. 2015; Huang et al. 2016)

450 suggest that gene expression is under strong stabilizing selection. Stabilizing selection on

451 gene expression predicts that mutational variance $\left(V_{m}\right)$ in transcript abundance is greatest

452 where the effects of genetic drift are predicted to be highest, such as in our $N=1 \mathrm{MA}$

453 lines. Our results are consistent with this prediction in that the $N=1 \mathrm{MA}$ lines exhibit

454 significantly greater mutational variation in transcript abundance relative to larger

455 population size treatments $(N=10,100 \mathrm{MA}$ lines). We have previously reported that

456 regulation of transposable element transcription is also under selection in these MA lines

457 and that TE transcription increased with time, especially in the $N=1$ lines (Bergthorsson

458 et al. 2020). Furthermore, gene copy-number variants that substantially increased

459 transcript abundance were less likely to accumulate in larger populations relative to the

460 MA lines subjected to the most extreme population bottleneck, which demonstrated the

461 importance of expression of gene duplicates for fitness (Konrad et al. 2018). In the

462 analyses presented here, TEs and CNVs were filtered out. The number of mutations per

463 generation that accumulated in these MA lines was not significantly different between the 
464 three population size treatments (Konrad et al. 2019), but it nevertheless appears that

465 mutations altering change gene expression are associated with reduced fitness, and have

466 been selected against in MA lines with larger population bottlenecks.

The residual variance, $V_{r}$, reflecting the within-line variation in gene expression

470 Furthermore, $V_{r}$ was significantly greater in the $N=1$ MA lines relative to their $N>1$

471 counterparts. As with the mutational variance, this suggests that mutations resulting in

472 increased sensitivity to environmental and transcriptional noise due to dysregulation of

473 gene expression are accumulating to the greatest degree under conditions of extreme

474 genetic drift, and to a lesser degree in MA lines with larger population bottlenecks (and

475 greater efficiency of selection). This increase in the $V_{r}$ for transcription in our MA lines

476 contrasts with a previous $C$. elegans study using DNA microarrays in which the ancestral

477 N2 control was found to have higher $V_{r}$ than the MA lines (Baer and Denver 2010). The

478 observation that an increase in $V_{r}$ largely mirrors the pattern for $V_{m}$ suggests that the two

479 may be related and perhaps stem from the same mechanistic causes.

There was a strong positive correlation between the per gene $V_{m}$ and $V_{r}$. This

482 correlation is in accord with results from other experimental systems such as $S$.

483 cerevisiae and D. melanogaster where mutational variation in gene expression was

484 determined to be positively associated with environmental variation and gene expression

485 noise (Rifkin et al. 2005; Landry et al. 2007; Huang et al. 2016). The canalization of

486 gene expression, which renders patterns of gene expression robust to the effects of

487 environmental variation, also appears to buffer the perturbing effects of mutations by

488 similar means (Wagner et al. 1997; Meiklejohn and Hartl 2002; Rifkin et al. 2005;

489 Landry et al. 2007; Baer and Denver 2010; Huang et al. 2016).

While the mutational variance $\left(V_{m}\right)$ in transcript abundance was negatively

492 correlated with population size, the $V_{m} / V_{r}$ ratio for transcript abundance unexpectedly

493 revealed the opposite relationship (smaller in the $N=1$ lines than in the $N>1$ MA lines).

494 This was owing to a disproportionate increase in residual variance, $V_{r}$, in the $N=1$ lines 
495 relative to the $N>1$ MA lines. Decanalization is often portrayed as increasing

496 evolvability by revealing cryptic genetic variation (Wagner et al. 1997; Gibson and

497 Dworkin 2004; Flatt 2005; Baer 2008). Conversely, if canalization makes phenotypes

498 robust to genetic and environmental perturbation by the same mechanisms,

499 decanalization may also decouple the genotype and phenotype by increasing

500 microenvironmental sensitivity and noise in gene expression to such a degree that natural

501 selection becomes less effective in the short-term. However, the fact that higher $V_{r}$ and a

502 lower $V_{m} / V_{r}$ ratio (predicted to be correlated with mutational heritability) are observed in

503 the $N=1$ lines suggests that decanalization of a large number of genes is itself often

504 deleterious, and would be selected against under realistic conditions in natural

505 populations.

We investigated the influence of chromatin domains on the variation in gene 508 expression utilizing five broad domain categories that have been previously defined (Liu 509 et al. 2011). The median CV of transcript abundance in the ancestral control of the MA

510 lines was very similar between genes in different domains. However, following mutation

511 accumulation, the median CV increased for genes in all five domains across all

512 population size treatments. The greatest and smallest increase in median CV was for

513 genes associated with broad gene silencing and active transcription, respectively. Within

514 each domain category, the $N=1$ lines always exhibited the greatest increase in median

$515 \mathrm{CV}$ for gene expression. For example, in repressed domains, the median $\mathrm{CV}$ in the $N=1$

516 lines increased by $65 \%$ compared to a $37 \%$ and $50 \%$ increase in the $N=10$ and $N=100$

517 lines, respectively. In constitutively active domains, the median CV in the $N=1$ lines

518 increased by $32 \%$ compared to a $13 \%$ and $6 \%$ increase in the $N>1$ lines $(N=10$ and $N=$

519 100). Genes in domains associated with silencing of repeats, dosage compensation and

520 activated transcription fell between these extremes. It appears that the regulation of genes

521 in repressed domains is most sensitive to genetic perturbation from the accumulation of

522 mutations.

524 Mutations can alter gene expression, thereby resulting in phenotypic divergence

525 from the wildtype. In terms of transcript abundance, this dysregulation of gene expression 
526 following mutation can manifest in two modes, namely (i) underexpression, or (ii)

527 overexpression. Both these deviations from wildtype expression levels have the potential

528 to alter phenotypes in a detrimental or beneficial manner, depending on the

529 environmental context. There are inherent challenges in our ability to predict if, and how,

530 a particular new mutation will influence gene expression, especially if it is trans-acting

531 (Lutz et al. 2019; Hill et al. 2020). Nevertheless, we sought to investigate if spontaneous

532 mutations confer any broad, general patterns with respect to expression dysregulation

533 under minimal and increasing intensity of selection. More genes were significantly

534 underexpressed than overexpressed after mutation accumulation across all population

535 size treatments. However, the ratio of significantly overexpressed to underexpressed

536 genes was greater in the $N=1$ MA lines. This pattern could be suggestive of a greater

537 deleterious fitness effect of gene overexpression and a role of purifying selection in the

538 eradication of such mutations at larger population sizes. Alternatively, the association

539 between genes with increased gene expression and population size could be a

540 consequence of the, on average, lower fitness of the $N=1$ lines (Katju et al. 2015, 2018).

541 Some alterations in gene expression could, for example, be compensating for mutations

542 causing transcriptional dysregulation of other genes.

544 An examination of gene expression profiles offers a direct approach to investigate

545 whether genes are equally malleable in their ability to diverge at the transcriptional level.

546 Genes associated with the mitochondrial membrane and immune system exhibited the

547 greatest average increase in expression over ancestral levels in the MA lines. The

548 vigorous upregulation of immune response genes was especially pronounced in low

549 fitness $(N=1)$ lines (Figure 5) despite the absence of a pathogenic challenge. While the

550 standard C. elegans practice of feeding nematodes live OP50 (E. coli) has been

551 documented to decrease lifespan compared to nematodes fed dead bacteria in a model of

552 slow pathogenesis (Battisti et al. 2016; Darby 2005), the L1 larvae sequenced for this

553 experiment were not exposed to food, living or dead, following synchronization.

554 Furthermore, while these $N=1$ lines possessed the greatest degree of upregulation in

555 downstream inflammatory response genes, we did not observe any significant expression

556 changes in key regulatory genes upregulated directly in response to bacterial challenge, 
557 such as pmk-1/pmk-2, sek-1, and nsy-1 (Kim et al. 2002). However, infection response

558 genes in C. elegans share significant overlap with stress response pathways and can be

559 triggered by cellular stress or damage (Matzinger 1994). Because immune effectors

560 increase ER stress and increased expression in the unfolded protein response (UPR)

561 pathway, another category predominantly enriched in the $N=1$ lines (Figure 5), we

562 turned focus on to the ire-1/xbp-1 pathway. This pathway has been documented to confer

563 host protection via alleviation of ER stress resulting from immune system activation

564 (Calfon et al. 2002; Shapira et al. 2006; Richardson et al. 2010). Only a single line, 1N,

565 was found to possess significant upregulation of genes within this network,

566 presumptively providing protection against the detrimental effect of increased

567 inflammation. Thus, the negative fitness effects brought about by voracious activation of

568 immune pathways might be coupled with increased ER stress and a lack of concurrent

569 increases in pathways involved in managing such stresses.

Previous analyses into the fitness effects of mutations in lines of C. elegans MA

572 lines with population bottlenecks of $N=1,10$ and 100 found no significant fitness

573 decline in the $N=10$, and 100 lines (Katju et al. 2015, 2018). Furthermore, whole-

574 genome sequencing found no differences in the accumulation of base substitutions and

575 small indels among the MA lines of different population sizes (Konrad et al. 2019). The

576 molecular differences that we have found between MA lines that were maintained under

577 different efficiency of selection (different population sizes) are primarily associated with

578 expression: increased expression and increased variation in expression of transposable

579 elements (Bergthorsson et al. 2020), greater expression of duplicated genes (Konrad et al.

580 2018), and greater variation of expression in other genes. It has been noted that the

581 number of genes with significant differential expression in MA lines is at least an order

582 of magnitude greater than the number of mutations per genome (Gibson 2005), which is

583 confirmed in this study. These results suggest that mutations that cause large-scale

584 changes in gene expression are more deleterious than those that alter gene expression to a

585 lesser degree. This is also in accord with our previous results regarding TEs and

586 expression of gene duplicates. However, some of the changes in gene expression in low

587 fitness lines may be attempts to ameliorate the effects of prior deleterious mutations. For 
588 example, the negative correlation between fitness and increased transcription of

589 immune/stress/unfolded protein response genes may be examples of compensatory

590 response in gene expression rather than contributing directly to fitness decline. Our study

591 extends previous findings regarding balancing selection on gene expression. Furthermore,

592 we also observed significant expression divergence in MA lines that did not exhibit

593 significant fitness loss. However, the population sizes used in these experiments are too

594 small to conclude that this expression divergence is neutral.

595

596 ACKNOWLEDGMENTS

597 This research was partially supported by National Science Foundation grant MCB-

5981565844 to V.K. U.B. and V.K. were additionally supported by start-up funds from the

599 Department of Veterinary Integrative Biosciences, College of Veterinary Medicine and

600 Biomedical Sciences at Texas A\&M University.

601

\section{AUTHOR CONTRIBUTIONS}

603 TCD, Acquisition of data, Analysis and interpretation of data, Drafting or revising the

604 article; AK, Acquisition of data, Analysis and interpretation of data; UB and VK,

605 Conception and design, Analysis and interpretation of data, Drafting or revising the

606 article.

608 AUTHOR ORCIDs

609 Ulfar Bergthorsson, http://orcid.org/0000-0003-1419-1349

610 Vaishali Katju, http://orcid.org/0000-0003-4720-9007

612 COMPETING INTERESTS

613 The authors declare that no competing interests exist.

615 ADDITIONAL FILES

616 Supplementary files

617 - Supplementary file 1. Supplemental Tables S1 and S2. 
618 - Supplementary file 2: Supplemental Figure S1

619 - Supplementary file 3: Supplemental Figure S1

\section{Major Datasets}

621 The sequence data generated in this study is available in Bioproject PRJNA448413 with

622 accession code SRP145329 (Bioproject link:

623 https://www.ncbi.nlm.nih.gov/bioproject/PRJNA448413). 


\section{LITERATURE CITED}

Abzhanov A, Protas M, Grant BR, Grant PR, Tabin CJ. 2004. Bmp4 and morphological variation of beaks in Darwin's finches. Science 305:1462-1465. DOI: 10.1126/science.1098095.

Baer CF. 2008. Quantifying the decanalizing effects of spontaneous mutations in Rhabditid nematodes. Am. Nat. 172: 272-281. DOI: 10.1086/589455

Baer CF, Denver DR. 2010. Spontaneous mutations decrease sensitivity of gene expression to random environmental variation in Caenorhabditis elegans. PLoS One 5:e8750. DOI: 10.1371/journal.pone.0008750.

Battisti JM, Watson LA, Naung MT, Drobish AM, Voronina E, Minnick MF. 2017. Analysis of the Caenorhabditis elegans innate immune response to Coxiella burnetii. Innate Immun. 23:111-127. DOI: 10.1177/1753425916679255.

Bergthorsson U, Sheeba CJ, Konrad A, Belicard T, Beltran T, Katju V, Sarkies P. 2020. Longterm experimental evolution reveals purifying selection on piRNA-mediated control of transposable element expression. BMC Biology 18:162. DOI: 10.1186/s12915-02000897-y.

Britten RJ, Davidson EH. 1969. Gene regulation for higher cells: a theory. Science 165:349-357. DOI: $10.1126 /$ science.165.3891.349.

Calfon M, Zeng H, Urano F, Till JH, Hubbard SR, Harding HP, Clark SG, Ron D. 2002. IRE1 couples endoplasmic reticulum load to secretory capacity by processing the XBP-1 mRNA. Nature 415:92-96. DOI: 10.1038/415092a.

Charlesworth B. 2009. Effective population size and patterns of molecular evolution and variation. Nat. Rev. Genet. 10:195-205. DOI: 10.1038/nrg2526.

Contrino S, Smith RN, Butano D, Carr A, Hu F, et al. 2012. modMine: flexible access to modENCODE data. Nucl. Acids Res. 40:D1082-D1088. DOI: 10.1093/nar/gkr921.

Crow JF, Kimura M, eds. 1970. An Introduction to Population Genetics Theory. Harper and Row, New York.

Darby C. 2005. Interactions with microbial pathogens. WormBook, pp. 1-15.

DOI: 10.1895/wormbook.1.21.1. http://www.wormbook.org.

Denver DR, Morris K, Streelman JT, Kim SK, Lynch M, Thomas WK. 2005. The transcriptional 
consequences of mutation and natural selection in Caenorhabditis elegans. Nat. Genet. 37:544-548. DOI: $10.1038 / \mathrm{ng} 1554$.

Dobin A, Davis CA, Schlesinger F, Drenkow J, Zaleski C, Jha S, Batut P, Chaisson M, Gingeras TR. 2013. STAR: ultrafast universal RNA-seq aligner. Bioinformatics 29:15-21. DOI: 10.1093/bioinformatics/bts635.

Fay JC, McCullough HL, Sniegowski PD, Eisen MB. 2004. Population genetic variation in gene expression is associated with phenotypic variation in Saccharomyces cerevisiae. Genome Biol. 5:R26. DOI: 10.1186/gb-2004-5-4-r26.

Fay JC, Wittkopp JC. 2008. Evaluating the role of natural selection in the evolution of gene regulation. Heredity 100:191-199. DOI: 10.1038/sj.hdy.6801000.

Flatt T. 2005. The evolutionary genetics of canalization. Q. Rev. Biol. 80:287-316. DOI: $10.1086 / 432265$.

Gibson G. 2005. Mutation accumulation of the transcriptome. Nat. Genet. 37:458-460. DOI: 10.1038/ng0505-458.

Gibson G, Dworkin I. 2004. Uncovering cryptic genetic variation. Nat. Rev. Genet. 5:681-690. DOI: $10.1038 / \operatorname{nrg} 1426$.

Gilad Y, Oshlack A, Rifkin SA. 2006. Natural selection on gene expression. Trends Genet. 22:456-461. DOI: 10.1016/j.tig.2006.06.002.

Gompel N, Prud'homme B, Wittkopp PJ, Kassner VA, Carroll SB. 2005. Chance caught on the wing: cis-regulatory and the origin of pigment patterns in Drosophila. Nature 433:481487. DOI: $10.1038 /$ nature03235.

Halligan DL, Keightley PD. 2009. Spontaneous mutation accumulation studies in evolutionary genetics. Annu. Rev. Ecol. Evol. Syst. 40:151-172. DOI:

10.1146/annurev.ecolsys.39.110707.173437.

Hill MS, Vande Zande P, Wittkopp PJ. 2021. Molecular and evolutionary processes generating variation in gene expression. Nat. Rev. Genet. 22:203-215. DOI: 10.1038/s41576020-00304-w.

Hodgins-Davis A, Rice DP, Townsend JP. 2015. Gene expression evolves under a house-ofcards model of stabilizing selection. Mol. Biol. Evol. 32:2130-2140. DOI: 10.1093/molbev/msv094. 
Houle D, Morikawa B, Lynch M. 1996. Comparing mutational variabilities. Genetics 143:14671483.

Huang W, Lyman RF, Lyman RA, Carbone MA, Harbison ST, Magwire MM, Mackay TF. 2016. Spontaneous mutations and the origin and maintenance of quantitative genetic variation. Elife 5:e14625. DOI: 10.7554/eLife.14625.

Hutter S, Saminadin-Peter SS, Stephan W, Parsch J. 2008. Gene expression variation in African and European populations of Drosophila melanogaster. Genome Biol. 9:R12. DOI: 10.1186/gb-2008-9-1-r12.

Katju V, Bergthorsson U. 2019. Old trade, new tricks: insights into the spontaneous mutation process from the partnering of classic mutation accumulation experiments with highthroughput genomic approaches. Genome Biol. Evol. 11:136-165. DOI: $10.1093 / \mathrm{gbe} / \mathrm{evy} 252$.

Katju V, Packard LB, Bu L, Keightley PD, Bergthorsson U. 2015. Fitness decline in spontaneous mutation accumulation lines of Caenorhabditis elegans with varying effective population sizes. Evolution 69:104-116. DOI: 10.1111/evo.12554.

Katju V, Packard LB, Keightley PD. 2018. Fitness decline under osmotic stress in Caenorhabditis elegans populations subjected to spontaneous mutation accumulation at varying population sizes. Evolution 72:1000-1008. DOI: 10.1111/evo.13463.

Kim DH, Feinbaum R, Alloing G, Emerson FE, Garsin DA, et al. 2002. A conserved p38 MAP kinase pathway in Caenorhabditis elegans innate immunity. Science 297:623-626. DOI: 10.1126/science. 1073759.

Kimura M. 1962. On the probability of fixation of mutant genes in a population. Genetics 47: $713-719$.

King MC, Wilson AC. 1975. Evolution at two levels in humans and chimpanzees. Science 188:107-116. DOI: 10.1126/science.1090005.

Konrad A, Brady MJ, Bergthorsson U, Katju V. 2019. Mutational landscape of spontaneous base substitutions and small indels in experimental Caenorhabditis elegans populations of differing size. Genetics 212:837-854. DOI: 10.1534/genetics.119.302054.

Konrad A, Flibotte S, Taylor J, Waterston RH, Moerman DG, Bergthorsson U, Katju V. 2018. Mutational and transcriptional landscape of spontaneous gene duplications and deletions 
in Caenorhabditis elegans. Proc. Natl. Acad. Sci. USA 115:7386-7391. DOI: 10.1073/pnas.1801930115.

Konrad A, Thompson O, Waterston RH, Moerman DG, Keightley PD, Bergthorsson U, Katju V. 2017. Mitochondrial mutation rate, spectrum and heteroplasmy in Caenorhabditis elegans spontaneous mutation accumulation lines of differing population size. Mol. Biol. Evol. 34:1319-1334. DOI: 10.1093/molbev/msx051.

Landry CR, Lemos B, Rifkin SA, Dickinson WJ, Hartl DL. 2007. Genetic properties influencing the evolvability of gene expression. Science 317:118-121. DOI:

10.1126/science. 1140247.

Lemos B, Meiklejohn CD, Cáceres M, Hartl DL. 2005. Rates of divergence in gene expression profiles of primates, mice, and flies: stabilizing selection and variability among functional categories. Evolution 59:126-137.

Liu T, Rechtsteiner A, Egelhofer TA, Vielle A, Latorre I, et al. 2011. Broad chromosomal domains of histone modification patterns in C. elegans. Genome Res. 21:227-236. DOI: 10.1101/gr.115519.110.

Loehlin DW, Ames JR, Vaccaro K, Carroll SB. 2019. A major role for noncoding regulatory mutations in the evolution of enzyme activity. Proc. Natl. Acad. Sci. USA 116:1238312389. DOI: 10.1073/pnas.1904071116.

Lutz S, Brion C, Kliebhan M, Albert FW. 2019. DNA variants affecting the expression of numerous genes in trans have diverse mechanisms of action and evolutionary histories. PLoS Genet. 15:e1008375. DOI: 10.1371/journal.pgen.1008375.

Lynch M. 1988. The rate of polygenic mutation. Genet. Res. 51:137-148. DOI: $10.1017 / \mathrm{s} 0016672300024150$.

Matzinger P. 1994. Tolerance, danger, and the extended family. Annu. Rev. Immunol. 12:9911045. DOI: 10.1146/annurev.iy.12.040194.005015.

McGuigan K, Collet JM, Allen SL, Chenoweth SF, Blows MW. 2014a. Pleiotropic mutations are subject to strong stabilizing selection. Genetics 197:1051-1062. DOI: 10.1534/genetics.114.165720.

McGuigan K, Collet JM, McGraw EA, Ye YH, Allen SL, Chenoweth SF, Blows MW. 2014 b. The nature and extent of mutational pleiotropy in gene expression of male Drosophila serrata. Genetics 196:911-921. DOI: 10.1534/genetics.114.161232. 
Pollak E. 1987. On the theory of partially inbreeding finite populations. I. Partial selfing. Genetics 117:353-360.

R Core Team. 2014. R: A language and environment for statistical computing. Vienna, Austria: R Foundation for Statistical Computing.

Richardson CE, Kooistra T, Kim DH. 2010. An essential role for XBP-1 in host protection against immune activation in C. elegans. Nature 463:1092-1095. DOI: 10.1038 /nature08762.

Rifkin SA, Houle D, Kim J, White KP. 2005. A mutation accumulation assay reveals a broad capacity for rapid evolution of gene expression. Nature 438:220-223. DOI: 10.1038/nature04114.

Ritchie ME, Phipson B, Wu D, Hu Y, Law CW, Shi W, Smyth GK. 2015. limma powers differential expression analyses for RNA-sequencing and microarray studies. Nucleic Acids Research. 43:e47. DOI: 10.1093/nar/gkv007.

Shapira M, Hamlin BJ, Rong J, Chen K, Ronen M, Tan MW. 2006. A conserved role for a GATA transcription factor in regulating epithelial innate immune responses. Proc. Natl. Acad. Sci. USA. 103:14086-14091. DOI: 10.1073/pnas.0603424103.

Shapiro MD, Marks ME, Peichel CL, Blackman BK, Nereng KS, Jónsson B, Schluter D, Kingsley DM. 2004. Genetic and developmental basis of evolutionary pelvic reduction in threespine sticklebacks. Nature 428:717-723. DOI: 10.1038/nature02415.

Smyth GK. 2004. Linear models and empirical Bayes methods for assessing differential expression in microarray experiments. Statistical Applications in Genetics and Molecular Biology. Vol 3: Iss. 1. DOI: https://doi.org/10.2202/1544-6115.1027.

Stern DL. 1998. A role of Ultrabithorax in morphological differences between Drosophila species. Nature 396:463-466. DOI: 10.1038/24863.

Yu G, Wang L-G, Han Y, He Q-Y. 2012. clusterProfiler: an R package for comparing biological themes among gene clusters. OMICS 16:284-287. DOI: 10.1089/omi.2011.0118.

Wagner GP, Booth G, Bagheri-Chaichian H. 1997. A population genetic theory of canalization. Evolution 51:329-347. DOI: 10.1111/j.1558-5646.1997.tb02420.x.

Wickham, Hadley. 2016. ggplot2: Elegant Graphics for Data Analysis. New York: SpringerVerlag.

Wittkopp PJ, Williams BL, Selegue JE, Carroll SB. 2003. Drosophila pigmentation evolution: 
divergent genotypes underlying convergent phenotypes. Proc. Natl. Acad. Sci. USA

100:1808-1813. DOI: 10.1073/pnas.0336368100.

Wray GA, Hahn MW, Abouheif E, Balhoff JP, Pizer M, Rockman MV, Romano LA. 2003. The evolution of transcriptional regulation in eukaryotes. Mol. Biol. Evol. 20:1377-1419.

DOI: $10.1093 / \mathrm{molbev} / \mathrm{msg} 140$.

Zalts H, Yanai I. 2017. Developmental constraints shape the evolution of the nematode middevelopmental transition. Nat. Ecol. Evol. 1:113. DOI: 10.1038/s41559-017-0113.

Zhang Y, Liu T, Meyer CA, Eeckhoute J, Johnson DS, Bernstein BE, Nusbaum C, Myers RM, Brown M, Li W, Liu XS. 2008. Model-based analysis of ChIP-Seq (MACS). Genome Biol. 9: R137. DOI: 10.1186/gb-2008-9-9-r137. 


\section{FIGURE LEGENDS}

Figure 1. Mutational variance $\left(V_{m}\right)$, residual variance $\left(V_{r}\right)$, and the relationship between $V_{m}$ and $V_{\boldsymbol{r}}$ as a function of population size. (A) $V_{m}$ shows a negative association with population size across the MA lines. The distributions displayed as violin plots with an internal boxplot indicative of the population median were found to be significantly different (Kruskal-Wallis: $\chi^{2}$ $\left.=447.82, p=5.71 \times 10^{-89}\right)$. Median $V_{m}$ values for the $N=1,10$ and 100 lines were 1.11, 0.89, and $0.85 \times 10^{-4}$, respectively. Grand median $\left(9.55 \times 10^{-5}\right)$ for all experimental groups is shown as a dashed red line. (B) Median $V_{r}$ values for $N=1,10,100$ and the ancestral control were 5.89, $3.92,4.59$, and 2.17, respectively. Each of the three MA population size treatments have significantly greater $V_{r}$ than the ancestral control (Dunn's test: $Z=43.13, p=0 ; Z=22.08, p=$ $4.64 \times 10^{-108} ; Z=29.82, p=2.97 \times 10^{-195}$ for $N=1, N=10$ and $N=100$ respectively). The $V_{r}$ of the $N=1 \mathrm{MA}$ lines was significantly greater than in the $N=10$, and 100 lines $(N=1 \mathrm{vs} . N=10$ : Dunn's test: $Z=21.05, p=1.85 \times 10^{-98} ; N=1$ vs. $\left.N=100: Z=13.31, p=1.28 \times 10^{-40}\right)$. The $V_{r}$ of $N=100$ lines was greater than that of the $N=10$ lines (Dunn's test: $Z=7.74, p=4.96 \times 10^{-}$ ${ }^{15}$ ). The grand median (4.41) for all MA lines across the three population size treatments is shown as a dashed red line. $(C)$ The correlation between $V_{m}$ and $V_{r}$ is show for $N=1$ (yellow), 10 (red), and 100 (purple) MA lines. The results from linear regression are displayed for each population at the bottom right of the panel.

Figure 2. Lower $V_{m} / V_{r}$ ratio in the $N=1$ lines that are under minimal selection efficacy. The $V_{m} / V_{r}$ displayed as a violin plot with a median-centered boxplot, was significantly different between the three population size treatments of $N=1,10$, and 100 (Kruskal-Wallis $\chi^{2}=1225.36$, $p=8.25 \times 10^{-267}$ ). The median $V_{m} / V_{r}$ for gene expression were $1.68,2.41$, and $1.76 \times 10^{-3}$ for the $N=1,10$, and 100 MA lines, respectively.

\section{Figure 3. Number of differentially expressed genes and the magnitude of change are} increased in lines governed by random genetic drift. MA lines comprising the $N=1,10,100$ population size treatments are displayed in yellow, red, and purple respectively. (A) The average number of genes per MA line replicate that have significantly elevated or reduced transcript abundance relative to their N2 ancestor $(p \leq 0.01)$. Only genes that display $>20 \%$ increase or 
decrease in transcript abundance are included. Upregulated and downregulated genes are displayed with a solid bar above zero and a hatched bar below zero, respectively. Error bars represent the \pm standard error for each population size treatment. (B) A volcano plot depicting genes with significantly elevated or reduced transcript abundance relative to the N2 ancestral control. The horizontal axis displays the change in relative transcript abundance as $\log _{2}$ of the difference from the ancestral line. The vertical axis represents the $\log _{10}$ of adjusted $p$-value for each gene.

\section{Figure 4. The proportion of upregulated and downregulated genes within specific GO} categories highlight greater upregulation in the $N=1$ lines relative to other population size treatments. The average proportion of significantly upregulated (top, solid bars) and downregulated (bottom, striped bars) genes per line ( \pm standard error) relative to the ancestral N2 control is displayed for select GO categories.

\section{Figure 5. The Coefficient of Variation (CV) for genes associated with specific chromatin states are significantly higher in lines governed by extreme genetic drift $(N=1)$. The} boxplot represents the distribution of $\mathrm{CV}$ for genes located in genome regions associated with the histone modifications indicated on the x-axis. Significant differences were found between population sizes $(N=1,10$, and 100 individuals) for all chromatin states (Kruskal-Wallis: H3K27me3, $\chi^{2}=154.35, p=3.04 \times 10^{-34}$; H3K9me1/me2/me3, $\chi^{2}=35.47, p=1.99 \times 10^{-8}$; H3K27me1, H4K20me1, $\chi^{2}=102.74, p=4.91 \times 10^{-23}$; H3K4me2/me3, H4K8ac, H4K16ac, H4K16ac, H4Ktetra-ac, HTZ-1, $\chi^{2}=186.93, p=2.57 \times 10^{-41}$; H3K79me1/me2/me3, H3K36me3, $\left.\chi^{2}=72.58, p=1.74 \times 10^{-16}\right)$. The differences in $\mathrm{CV}$ for individual genes within the three different population size treatments are highly significant between chromatin states (Kruskal-Wallis: $N=$ $1, \chi^{2}=648.00, p=6.31 \times 10^{-139} ; N=10, \chi^{2}=628.78, p=9.15 \times 10^{-135} ; N=100, \chi^{2}=635.84, p=$ $\left.2.70 \times 10^{-136}\right)$. 
Figure 1

A

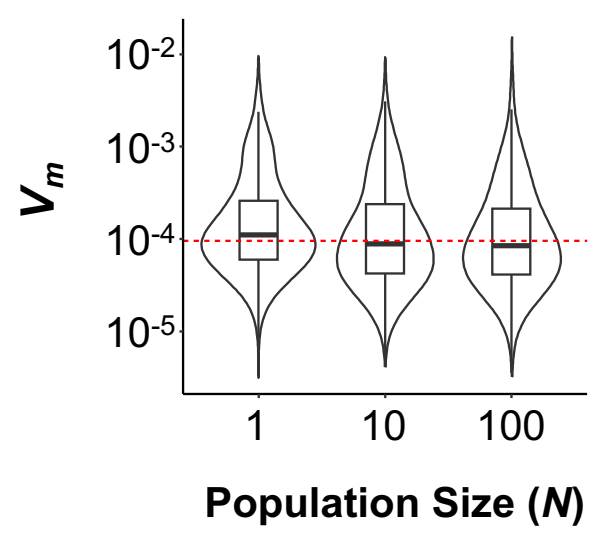

B

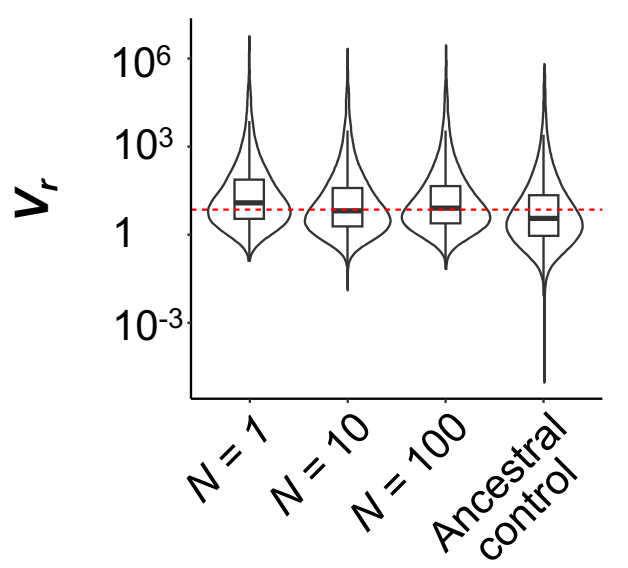

C

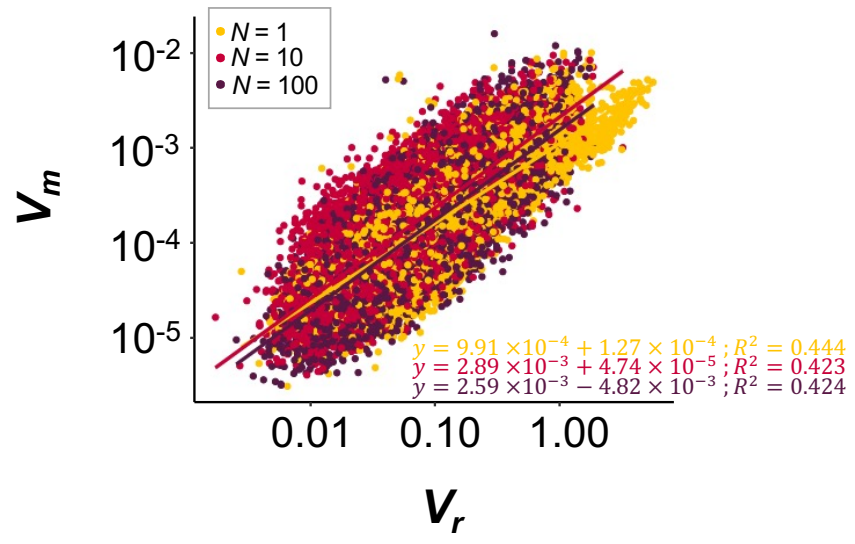


Figure 2

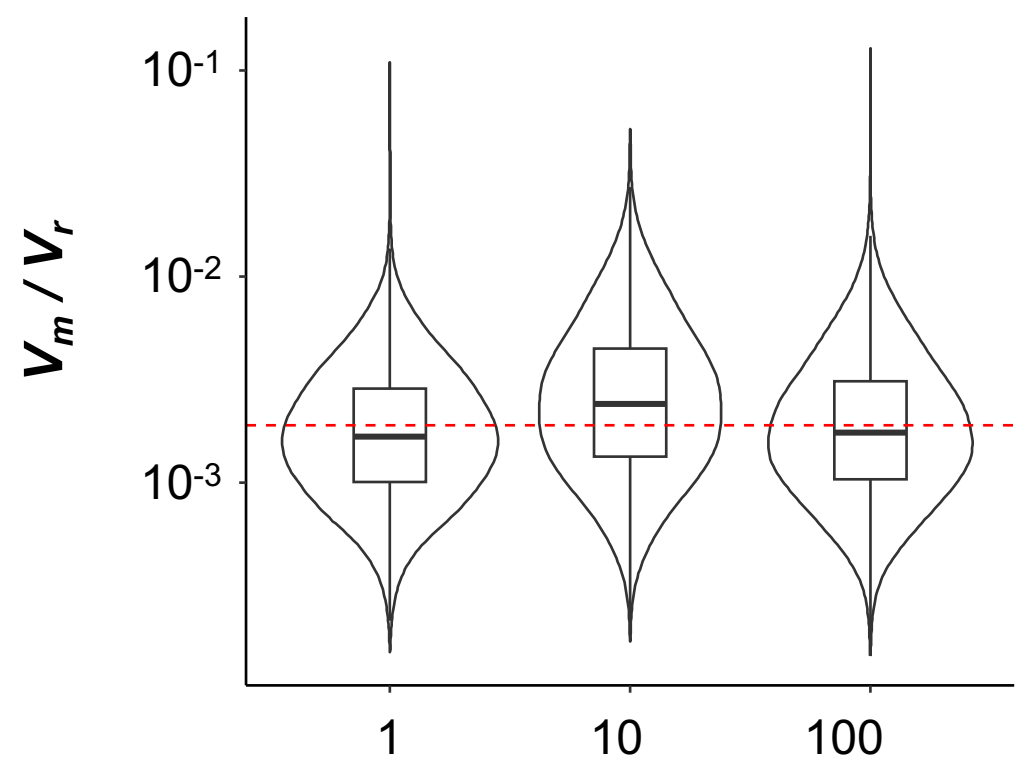

\section{Population Size $(N)$}




\section{Figure 3}
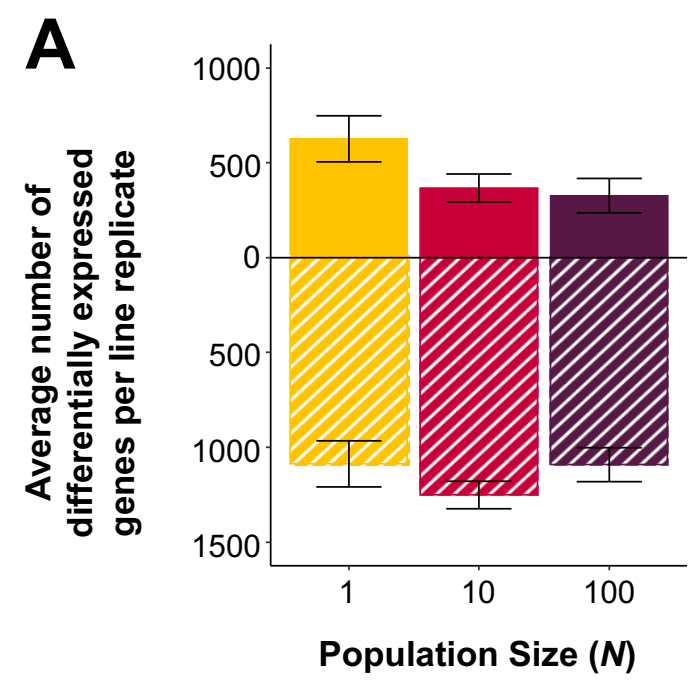

B

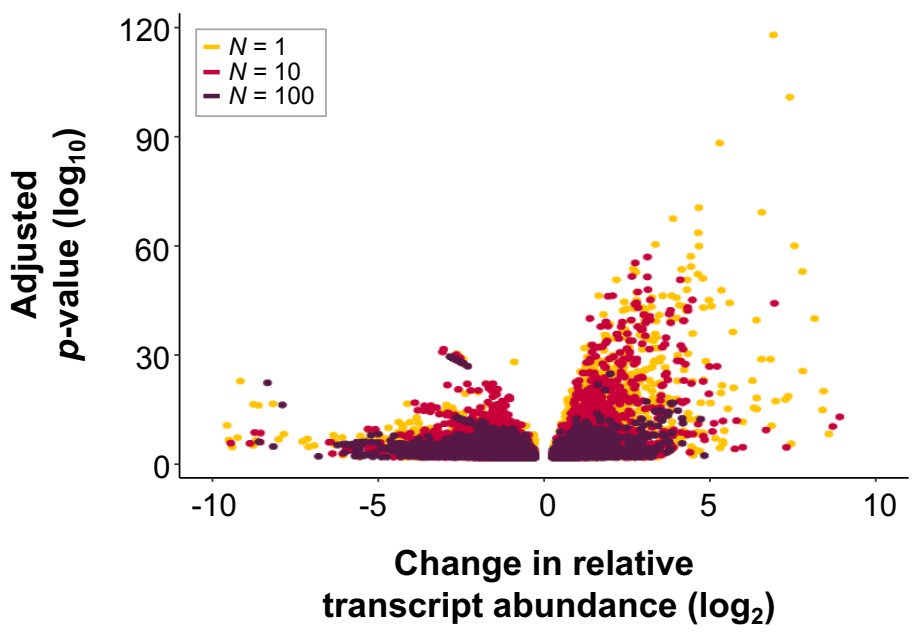


Figure 4

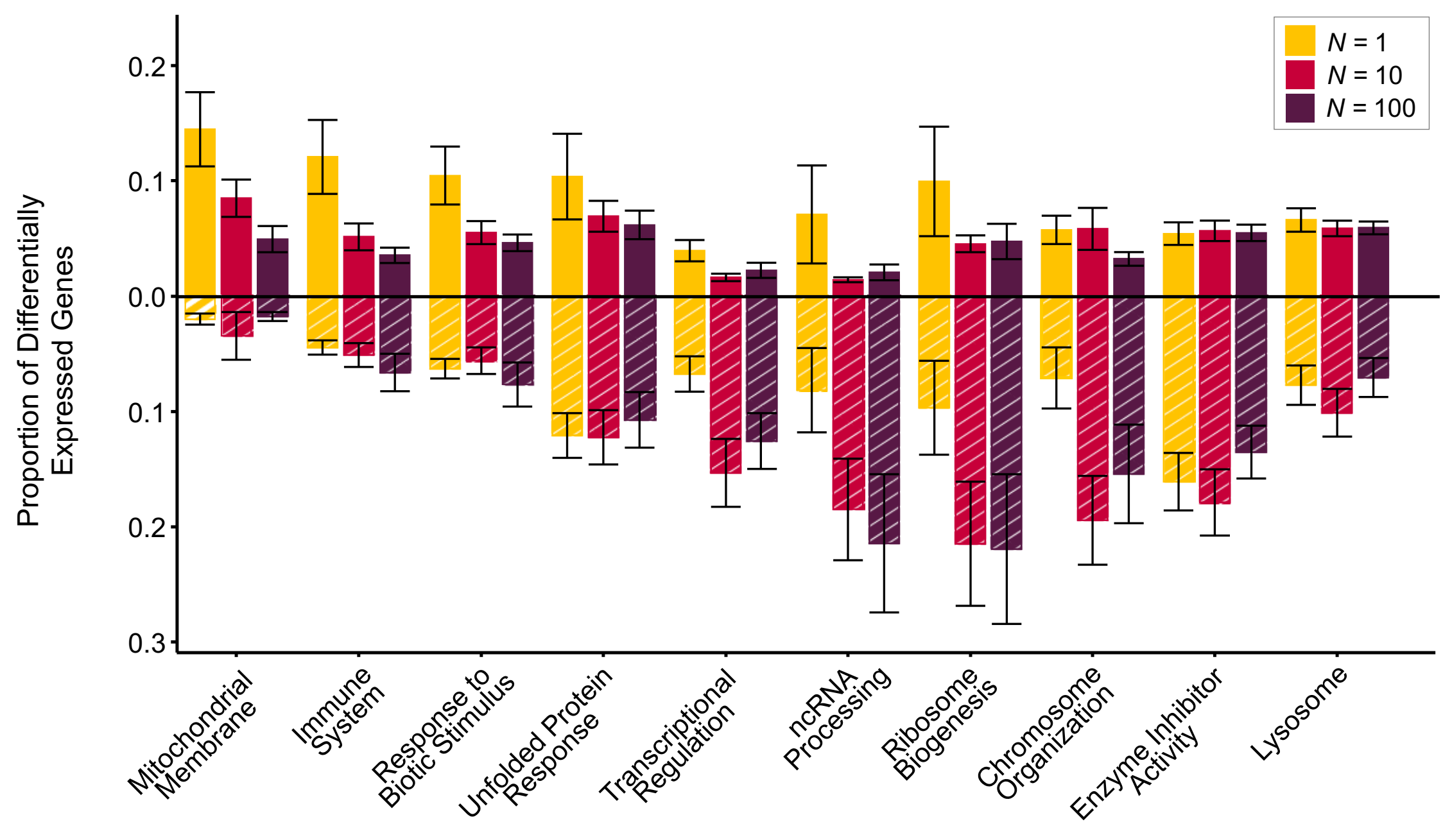




\section{Figure 5}

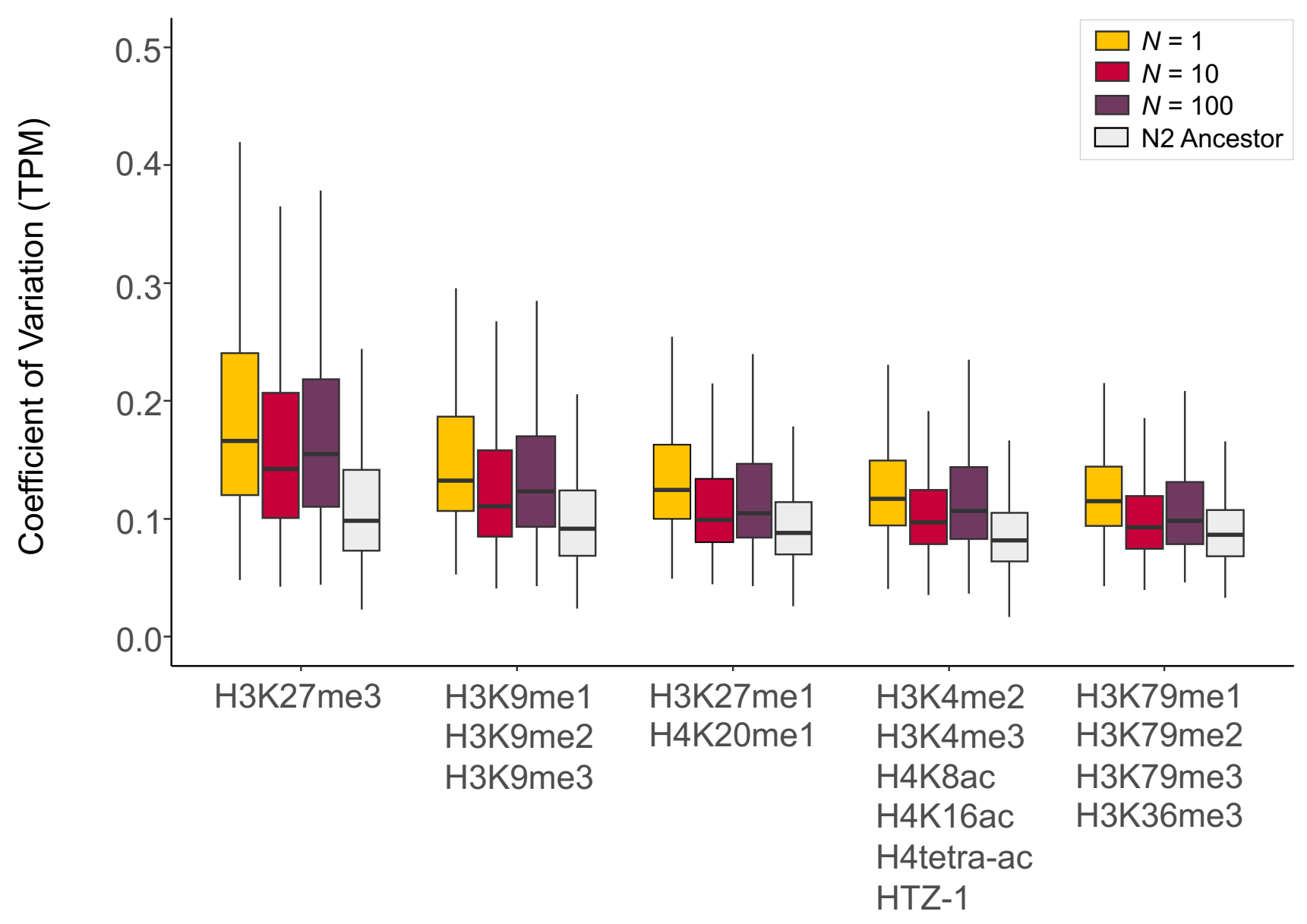

